\title{
A Clinico-Epidemiological Study of Acne in Adults
}

\author{
Swathi $\mathbf{G}^{1}$, Mamatha S Kusagur ${ }^{2}$ \\ ${ }^{1}$ Post Graduate, JJM Medical College, Davanagere \\ ${ }^{2}$ Associate Professor, Department of Dermatology, JJM Medical College, Davanagere
}

\begin{abstract}
Introduction: Acne is commonly viewed as a disorder of adolescence, however prevalence of adults with acne is increasing. Adult acne has been traditional defined as presence of acne beyond the age of 25 years. Aim: This study was designed to evaluate the clinical and epidemiological data of adult acne with respect to its special characteristics, aggravating and relieving factors. Design of the study: This is a prospective study Materials and methods: The study was carried out for 6 months and it included 50 patients. Patients above the age of 25years presenting with acne were included in the study. Results: Majority of the patients had grade-II acne 80\% followed by grade-I acne 10\%. The most common site of involvement was cheeks $80 \%$ followed by chin $66 \%$, mandibular-area $52 \%$ and forehead 48\%.Acne persisting beyond the age of 25years was observed in $70 \%$ while late-onset acne was seen in $30 \%$. Premenstrual flare was observed in 46\%, androgenetic-alopecia in 14\%. Scarring was observed in $46 \%$ and pigmentation in $30 \%$ of patients. Conclusion: Adult acne differs from adolescent acne by its more inflammatory nature and predominant involvement of cheeks and lower half of the face especially jaw-line and chin.
\end{abstract}

Keywords: Adult acne, Persistent acne

\section{Introduction}

Acne vulgaris is a common disorder affecting all ages and ethnic groups. Acne is a chronic inflammatory disorder of the pilosebaceous units characterised by seborrhoea, open and closed comedones, papules, pustules and in more severe cases nodules and pseudocysts.

The pathogenesis is attributed to multiple factors such as increased sebum production, follicular hyperkeratinization, proliferation of propionibacterium acne within the follicle, androgen activity, inflammation and immunological host reaction. ${ }^{1}$ Although acne is widely considered as a disorder of adolescence, prevalence of adults with acne is increasing. Adult acne has been traditionally defined as presence of acne beyond the age of 25 years. There are two types of adult acne; Persistent acne and late-onset acne. Adolescent acne persisting beyond the age of 25 years is called persistent adult acne and acne developing for the first time after the age of 25 years is called late-onset adult acne.

Acne vulgaris was graded using a simple grading system.

Grade 1-comedones, occasional papules

Grade 2- papules, comedones, few pustules

Grade 3- predominant pustules, nodules, abscess

Grade 4- mainly cysts, abscess, widespread scarring

We undertook this study to observe the prevalence, clinical features, and aggravating factors of acne in adults and highlight clear differences from adolescent acne. ${ }^{2}$

\section{Materials and Methods}

Study design: Prospective study of 50 patients with adult acne attending department of dermatology were included in the study. Study was done over a period of six months.

Inclusion criteria: Patients above the age of 25 years with acne of all grades.
Exclusion criteria: Patients who are taking oral contraceptive pills or any form of hormonal therapy, immune-compromised patients, pregnant and lactating women were excluded from the study.

A detailed history regarding the age, sex, occupation, and residence, duration of the disease, age of onset, medical and family history was obtained. A thorough dermatological examination was made to note the extent, site and type of lesions. Aggravating factors including application of cosmetics, topical steroids, drug intake, stress, premenstrual flare, seasonal variation, dandruff, diet, and smoking were noted. Markers of androgenicity such as hirsutism, alopecia were also included in the study. Written consent was obtained from all the patients.

\section{Results}

Out of 50 patients included in the study $92 \%$ (46) were women and $8 \%(4)$ were men. The age of the patients varied from 26 to 45 years with mean age of 30.1 years\{Fig:3\}. The duration of acne ranged from one week to 5 years. Persistent acne was observed in 70\%(35) while late-onset acne was observed in 30\%(15). Majority of the patients had grade-II acne $80 \%(40)\{$ Fig: 1$\}$ followed by grade-I 10\%(5), grade-III 8\%(4)\{Fig:2\} and grade-IV 2\%(1)\{Fig:4\}. The most common site of involvement was cheeks $80 \%$ followed by chin $66 \%$, mandibular area $52 \%$, forehead $48 \%$ and nose $16 \%$. Truncal involvement was rare and was seen in only $6 \%$ of the patients. Nature of the skin was dry in $54 \%$ and oily in $46 \%$. Diet was vegetarian in $54 \%$ and mixed in $46 \%$. Seborrhoeic dermatitis was the most common association found with acne vulgaris (34\%).Aggravation of acne due to stress was seen in $20 \%$ and with topical steroid usage in $12 \%$.Premenstrual flare was observed in $46 \%(23)$, androgenetic-alopecia in $14 \%$ and hirsutism in $10 \%$ of the patients. External factors such as residence, occupation, seasonal variation, drugs, cosmetics were not found to be significant aetiological factors. Scarring was observed in $46 \%$ and post acne pigmentation in $30 \%$ of patients. 


\section{International Journal of Science and Research (IJSR) \\ ISSN (Online): 2319-7064}

Index Copernicus Value (2013): 6.14 | Impact Factor (2014): 5.611

\section{Discussion}

In our study adult acne was observed to be more common in women $(92 \%)$. This is in concordance with the studies of Goulden et $\mathrm{al}^{3}$, and Khunger et $\mathrm{al}^{2}$, where $76 \%$ and $82.1 \%$ of the women were affected respectively. Our study noted persistent acne in $70 \%$ of the patients. In comparison Goulden et al., reported persistent acne in $82 \%$ and Khunger et al., reported in $73.2 \%$ of patients.

Our study revealed that majority of the patients with adult acne presented as grade II acne (80\%) with inflammatory papules and pustules followed by grade I acne $10 \%$. Comedonal acne was rarely observed in adult acne as compared with adolescent acne. Similar results were observed by Goulden et $\mathrm{al}^{3}$., where postadolescent acne was predominantly inflammatory.

In our study the most common site of involvement was cheeks $80 \%$ followed by chin $66 \%$ and mandibular area $52 \%$. Similar distribution was observed by Khunger et al. ${ }^{2}$, where cheeks was the most common site of involvement. This is in contrast to study by Capitanio et al. ${ }^{4}$, who have reported lower face and mandibular area as more common sites of involvement in post adolescent acne.

We observed premenstrual flare in $46 \%$ of the patients. Similar results were observed by Stoll et al. ${ }^{5}$, with premenstrual flare of $44 \%$. In the study by Goulden et al., $37 \%$ of women had at least one feature of hyperandrogenism as hirsutism (24.2\%), alopecia $(7.2 \%)$ or menstrual disturbance(17.7\%). In our study alopecia was observed in $14 \%$, hirsutism in $10 \%$ and menstrual disturbance in $6 \%$.

Aggravating factors such as stress in $20 \%$ and topical steroid usage in $12 \%$ of the cases was observed. Similarly Khunger et al., reported stress in $25.7 \%$ and steroid in $11.8 \%$ cases as aggravating factors. External factors such as residence, occupation, seasonal variation, exposure to sunlight, drugs, cosmetics were not found to be significant aetiological factors.
Our study observed scarring in $46 \%$ and post acne pigmentation in $30 \%$ of the cases. Khunger et al., observed scarring in $76.4 \%$ of the patients. A study by Adityan et al. ${ }^{6}$, on acne of all age groups documented scarring and hyperpigmentation in $39.5 \%$ and $24.6 \%$ of patients respectively.

\section{Conclusion}

Acne is the most common skin disease in dermatological practice and is the easiest skin condition to diagnose because of its distinct clinical features. Acne in adult women has significant psychosocial comorbidity and may be challenging to treat. Post adolescent acne mainly affects females. Adult acne differs from adolescent acne by its more inflammatory nature and predominant involvement of cheeks and lower half of the face especially jaw-line and chin. Facial scarring is more common which emphasizes the importance of adequate therapy.

\section{References}

[1] Durai PT, Nair DG.Acne vulgaris and quality of life among young adults in South India. Indian J Dermatol 2015;60:30-40.

[2] Khunger N, Kumar C. A clinic-epidemiological study of adult acne: is it different from adolescent acne? Indian $\mathrm{J}$ Dermatol Venereol Leprol 2012; 78(3):335-341.

[3] Goulden V, Clark S, Cunliffe W. Post- adolescent acne: A review of clinical features. Br J Dermatol 1997; 136:66- 70 .

[4] Capitanio B, Sinagra JL, Bordignon V, Fei PC, Picardo M, Zouboulis CC. Underestimated clinical features of postadolescent acne. J Am Acad Dermatol 2010; 63:782- 8 .

[5] Stoll S, Shalita AR, Webster GF, et al. The effect of the menstrual cycle on acne. J Am Acad Dermatol. 2001; 45(6):957-960.

[6] Adityan B, Thappa DM. Profile of acne vulgaris- A hospital- based study from South India. Indian J Dermatol Venereol Leprol 2009;75: 272- 8. 


\section{International Journal of Science and Research (IJSR)}

ISSN (Online): 2319-7064

Index Copernicus Value (2013): 6.14 | Impact Factor (2014): 5.611
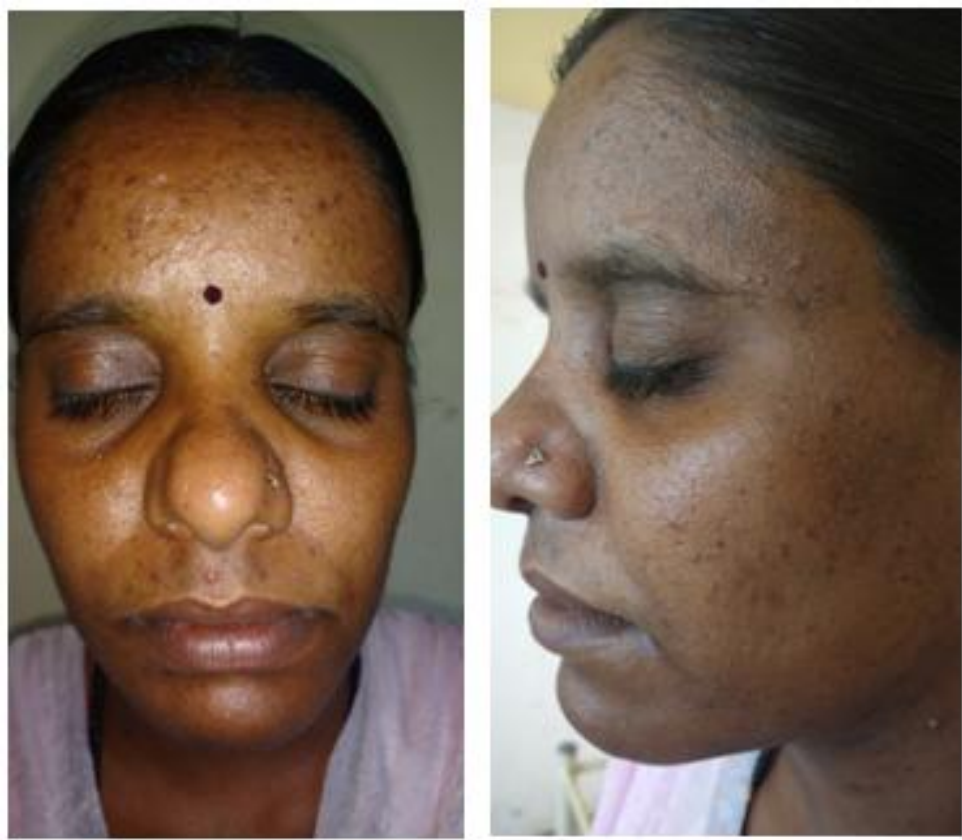

Figure 1: Grade II acne with predominant papules and hyperpigmentation in forehead, cheeks, mandibular area and chin
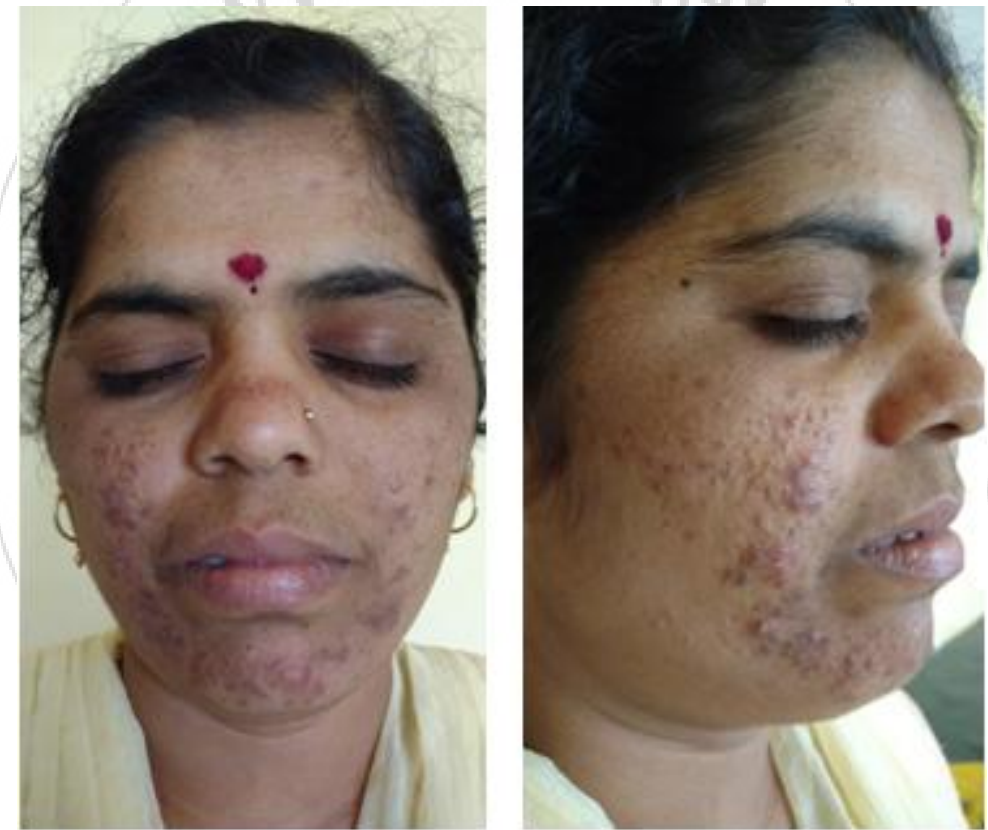

Figure 2: Grade III acne with inflammatory papules, pustules and few nodules in cheeks and chin.

Volume 4 Issue 11, November 2015 www.ijsr.net 


\section{FIGURE 3. AGE WISE DISTRIBUTION OF PATIENTS WITH ADULT ACNE}

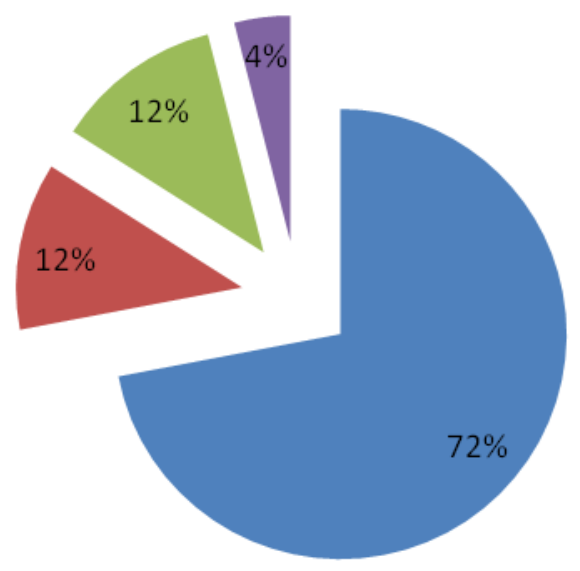

FIGURE 4. GRADING OF ADULT ACNE IN THE STUDY

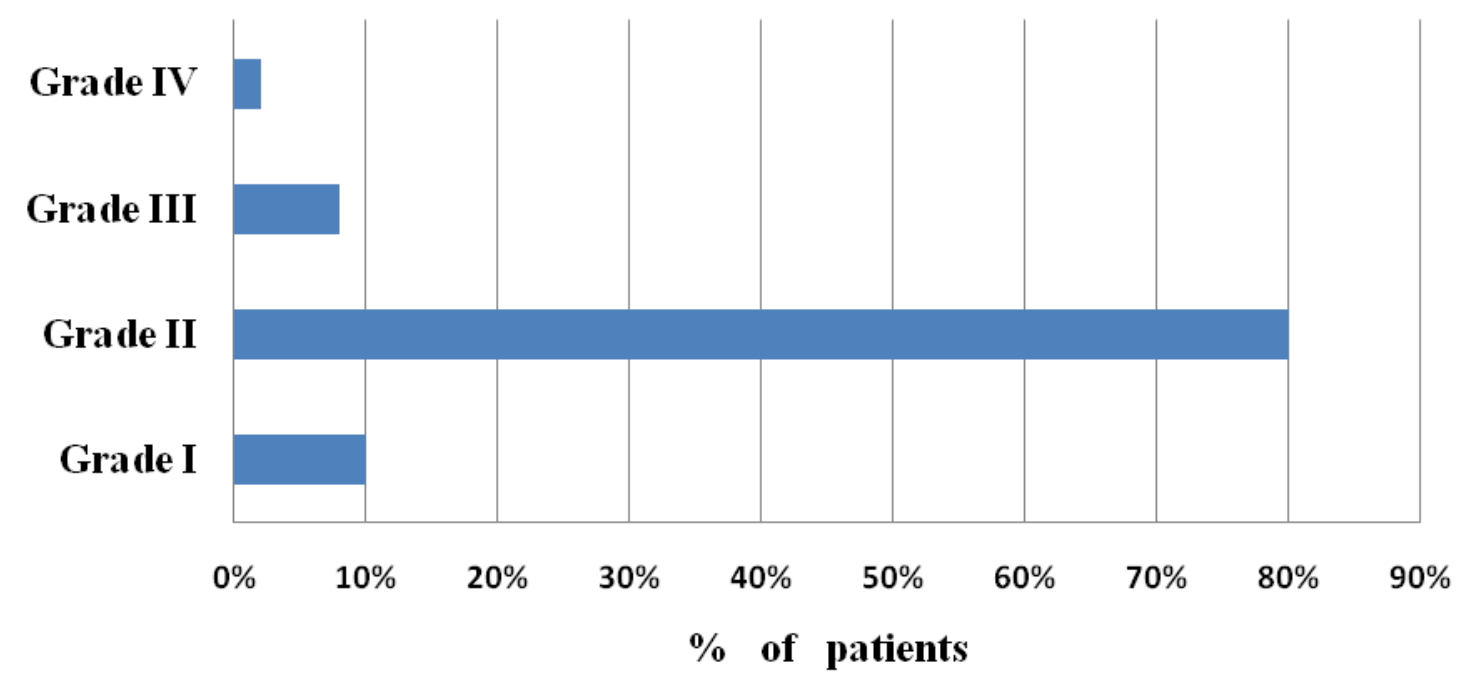

Volume 4 Issue 11, November 2015 